\title{
Preisgestaltung für Software-as-a-Service
}

\section{Ergebnisse einer empirischen Analyse mit Fokus auf nut- zungsabhängige Preismodelle}

\author{
Sonja Lehmann', Tobias Draisbach', Corina Koll', Peter Buxmann', \\ Heiner Diefenbach ${ }^{2}$ \\ ${ }^{1}$ Fachgebiet Information Systems, TU Darmstadt \\ ${ }^{2} \mathrm{TDS} A G$, Neckarsulm
}

\section{Einleitung}

Software wird heute zunehmend auch als Dienstleistung angeboten. Hierbei stellen Software- und Serviceanbieter Kunden Softwarelösungen via Internet zur Verfügung. Bei solchen Software-as-a-Service (SaaS)-Lösungen liegt die Verantwortung für Betrieb und Wartung der Software beim Anbieter (Cusumano 2007, S. 20; Buxmann et al. 2008b, S. 500). Kunden verbinden mit SaaS häufig den Wunsch nach flexiblen, ihren Bedürfnissen angepassten Preismodellen (Benlian et al. 2009). Daher stehen SaaS-Anbieter vor der Herausforderung, neue geeignete Preiskonzepte für diesen Markt zu entwickeln. Dabei besteht eine Grundsatzentscheidung darin, ob die Bepreisung nutzungsabhängig oder -unabhängig durchgeführt werden soll.

Vor diesem Hintergrund soll im vorliegenden Beitrag ein Überblick über derzeitig verwendete Preismodelle von SaaS-Anbietern gegeben werden. Hierzu wurden 114 SaaS-Anwendungen von 80 Anbietern anhand der Informationen ihrer Webseiten analysiert. Insbesondere soll die häufig in der Literatur vertretene These, dass nutzungsabhängige Preismodelle für $\mathrm{SaaS}$ grundsätzlich gut geeignet sind (siehe z. B. Kittlaus und Clough 2009, S. 59; Choudhary 2007), näher untersucht werden.

Im zweiten Kapitel wird zunächst ein Überblick über die Parameter der Software-Preisgestaltung gegeben. Diese Systematik dient als Grundlage für unsere empirische Analyse, deren wesentlichen Ergebnisse wir in Kapitel 3 vorstellen. Gegenstand des vierten Kapitels ist eine Fallstudie zur Untersuchung der Eignung einer nutzungsabhängigen und -unabhängigen Bepreisung von SaaS-Angeboten. Der Artikel schließt mit einem Fazit und einem Ausblick auf zukünftigen Forschungsbedarf. 


\section{Parameter der Software-Preisgestaltung}

Wie auch in anderen Branchen, sind die Möglichkeiten der Preisgestaltung in der Softwareindustrie vielfältig. Es gibt kein allgemeingültiges Preismodell für Softwareprodukte (Bontis und Chung 2000, S. 246), da sich Preismodelle aus verschiedenen Elementen zusammensetzen.

Um einen systematischen Überblick über mögliche Parameter der Softwarepreisgestaltung zu erhalten, verwenden wir folgende Gliederung der Softwarepreisgestaltung (Lehmann und Buxmann 2009):

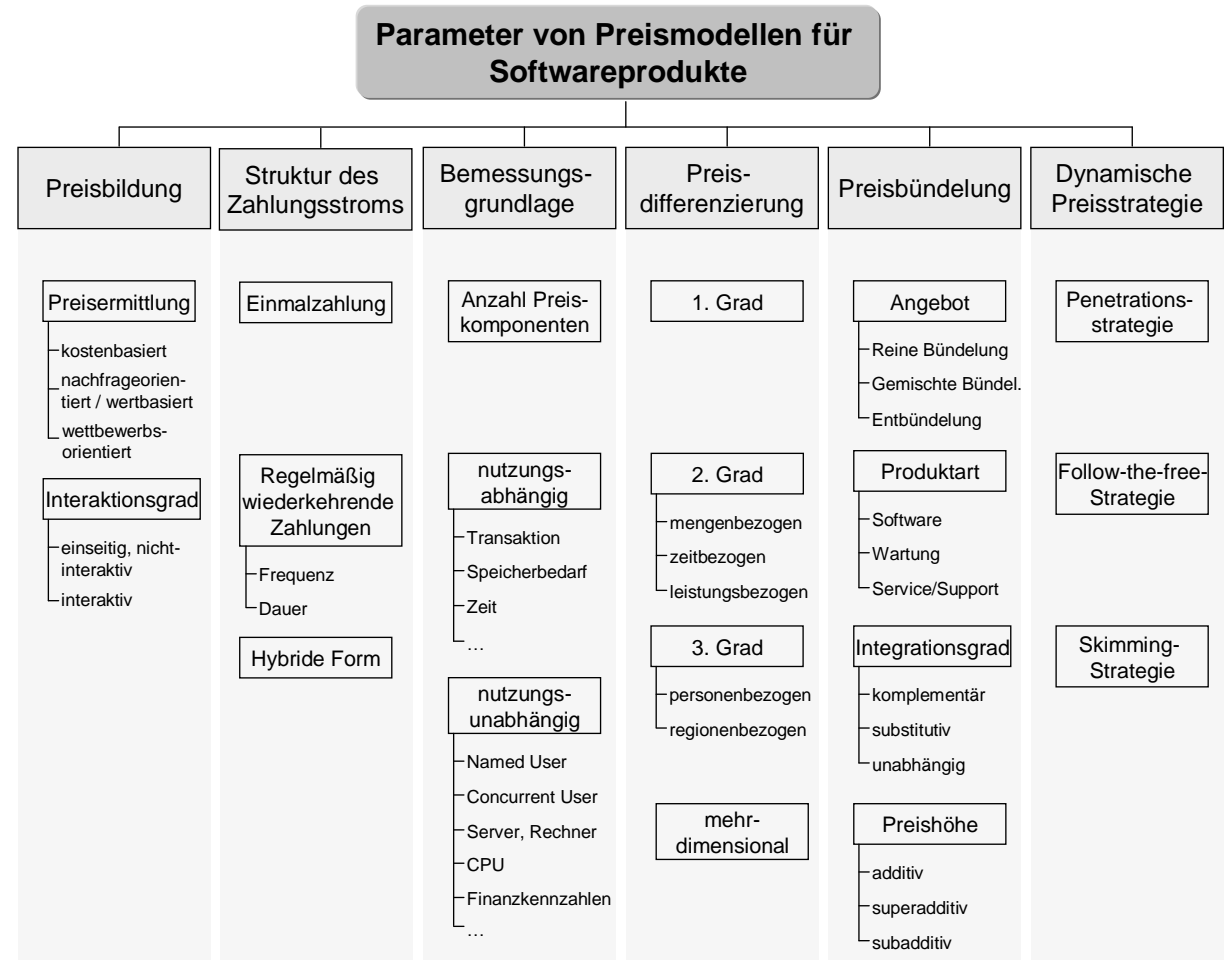

\section{Abbildung 1: Parameter der Software-Preisgestaltung (Lehmann und Buxmann 2009)}

Bei der Gestaltung eines Preismodells verwenden Softwareanbieter i. d. R. eine Kombination verschiedener Parameter in unterschiedlichen Ausprägungen.

Als Grundlage der Studie zur Untersuchung der Preismodelle im SaaS-Umfeld dient die oben dargestellte Systematisierung. ${ }^{1}$ Von den dort aufgeführten sechs Parametern wurden die Struktur des Zahlungsstroms, die Bemessungsgrundlage und die Preisdifferenzierung untersucht.

${ }^{1}$ Für eine ausführliche Darstellung siehe Lehmann und Buxmann 2009. 
Nicht betrachtet wurde der Parameter Preisbildung, da der Prozess der Preisbildung intern beim Anbieter erfolgt und i. d. R. nicht nach außen kommuniziert wird. Daher sind diesbezüglich auch keine Informationen auf öffentlichen Webseiten der Anbieter zu finden. Weiterhin wurde auch die Preisbündelung aus der Betrachtung herausgenommen. Hintergrund war hier die schwierige Zurechnung von Einzel- und Bündelprodukten, da es sich bei SaaS-Produkten per se um ein Bündel verschiedener Leistungen handelt (Cusumano 2007, S. 20). Aufgrund der Zeitpunktbetrachtung der Studie konnten dynamische Preisstrategien, die sich über einen längeren Zeitraum erstrecken, nicht analysiert werden.

\section{Status quo der SaaS-Preisgestaltung - ausgewählte Ergebnisse}

\subsection{Methodik und Datenbasis}

Ziel unserer empirischen Studie ist die Erhebung des Status quo derzeitiger SaaSAngebote in Bezug auf ihre aktuell verwendeten Preismodelle. Im Rahmen einer Inhaltsanalyse wurden im Zeitraum Dezember 2008 bis Februar 2009 die Webseiten von SaaS-Anbietern hinsichtlich ihrer Preismodelle für SaaS-Produkte untersucht. Dabei wurden neben Preislisten und sonstigen Preisangaben auch Dokumente über die Allgemeinen Geschäftsbedingungen (AGB) ausgewertet.

Als Ausgangspunkt für die Suche nach SaaS-Anbietern diente das „SaaSForum" (http://www.saas-forum.net). Dieses Informationsportal listet SaaSAnbieter auf, die ihre Produkte für den deutschsprachigen Markt anbieten. Derzeit sind mehr als 150 SaaS-Anwendungen auf dieser Seite aufgeführt.

Zielgruppe der Studie waren Anbieter mit SaaS-Produkten für Geschäftskunden, sodass 114 SaaS-Produkte von insgesamt 80 Anbietern analysiert werden konnten. Die Repräsentativität der betrachteten SaaS-Anbieter für den deutschsprachigen Markt kann nicht garantiert werden. Da wir jedoch alle Anbieter aus der SaaSForumsliste für den B2B-Bereich berücksichtigt haben und das „SaaS-Forum“ nach Kenntnis der Autoren derzeit die umfassendste Auflistung von SaaSAnbietern für den deutschsprachigen Markt ist, gehen wir davon aus, dass die untersuchte Stichprobe als für den Markt charakteristisch angesehen werden kann. Die Liste wird regelmäßig durch den Betreiber der Seite sowie durch eigenständige Registrierung seitens der SaaS-Anbieter aktualisiert und erweitert.

Hinsichtlich der Unternehmensgröße besteht die Stichprobe zu 53 Prozent aus SaaS-Anbietern mit bis zu 50 Mitarbeitern. Rund 8 Prozent der Anbieter haben zwischen 51 und 100 Mitarbeiter und 20 Prozent verfügen über mehr als 100 Mitarbeiter. Zu den größten SaaS-Anbietern, die in der Studie untersucht wurden, zählen Microsoft, Salesforce.com und Google.

Nach der Produktart wurden die SaaS-Anwendungen in die Kategorien „Datenmanagement“ (z. B. Content-Management-Systeme), „Geschäftsanwendung“ 
(z. B. ERP, CRM, Finanzanwendungen, eCommerce- und eProcurementlösungen), „Kommunikation \& Projektmanagement“ (z. B. Web Conferencing, Projektmanagementlösungen) sowie „Weitere“ (alle übrigen Produktarten) eingeteilt. Unter den betrachteten 114 SaaS-Produkten konnten 59 Produkte den Geschäftsanwendungen, 28 dem Bereich Kommunikation und Projektmanagement, 10 dem Datenmanagement und die verbleibenden 17 Produkte der Kategorie „Weitere“ zugeordnet werden.

Im Folgenden gehen wir auf ausgewählte Ergebnisse der Studie in Bezug auf die Preismodelle der Anbieter ein.

\subsection{Ergebnisse}

Die Analyse der jeweiligen Webseiten der Anbieter einschließlich der AGBs ergab ein gemischtes Bild über die Transparenz der Preismodelle.

Bei etwa einem Viertel der untersuchten SaaS-Produkte (26 Prozent) waren der Webseite des Anbieters keine Preisinformationen zu entnehmen. Unvollständige Informationen über das Preismodell der jeweiligen Lösung wurden bei ca. einem weiteren Viertel (26 Prozent) festgestellt. Beispielsweise gab es in diesen Fällen lediglich Aussagen zur Struktur des Zahlungsstroms. Bei knapp der Hälfte der betrachteten Anwendungen (48 Prozent) war das Preismodell einsehbar.

Das Kapitel ist im Weiteren nach der Systematik in Kapitel 2 gegliedert. Zunächst wird die Struktur des Zahlungsstroms betrachtet, bevor auf die Bemessungsgrundlage und die Preisdifferenzierung eingegangen wird. Die Untersuchung konzentriert sich im Folgenden auf die 84 SaaS-Produkte der Stichprobe, über die entweder teilweise oder vollständig Preisinformationen auf der Website des Anbieters vorliegen.

\section{Struktur des Zablungsstroms}

Die Struktur des Zahlungsstroms unterscheidet in Einmalzahlungen mit einem unbegrenzten Nutzungsrecht für den Kunden und regelmäßig wiederkehrende Zahlungen. Weiterhin ist auch eine hybride Form aus einmaligen und regelmäßigen Zahlungen denkbar. Die Analyse des Zahlungsstroms wird in Abbildung 2 dargestellt.

Die Ergebnisse zeigen, dass kein Produkt mit einer ausschließlichen Einmalzahlung angeboten wird. Allerdings werden in einigen Fällen Einmalzahlungen, wie z. B. Einrichtungsgebühren, in Kombination mit regelmäßigen Zahlungen verbunden.

Bezogen auf die SaaS-Produkte mit Informationen über die Struktur des Zahlungsstroms $(\mathrm{n}=78)$ ist die monatliche Zahlung mit 48 Produkten die am häufigsten eingesetzte Frequenz der Zahlung. Mit deutlichem Abstand folgt die jährliche Zahlung. Hinweise zur Mindestlaufzeit wurden bei 35 Produkten gefunden - mit 19 Nennungen ist eine zwölfmonatige Mindestdauer am häufigsten vertreten. 


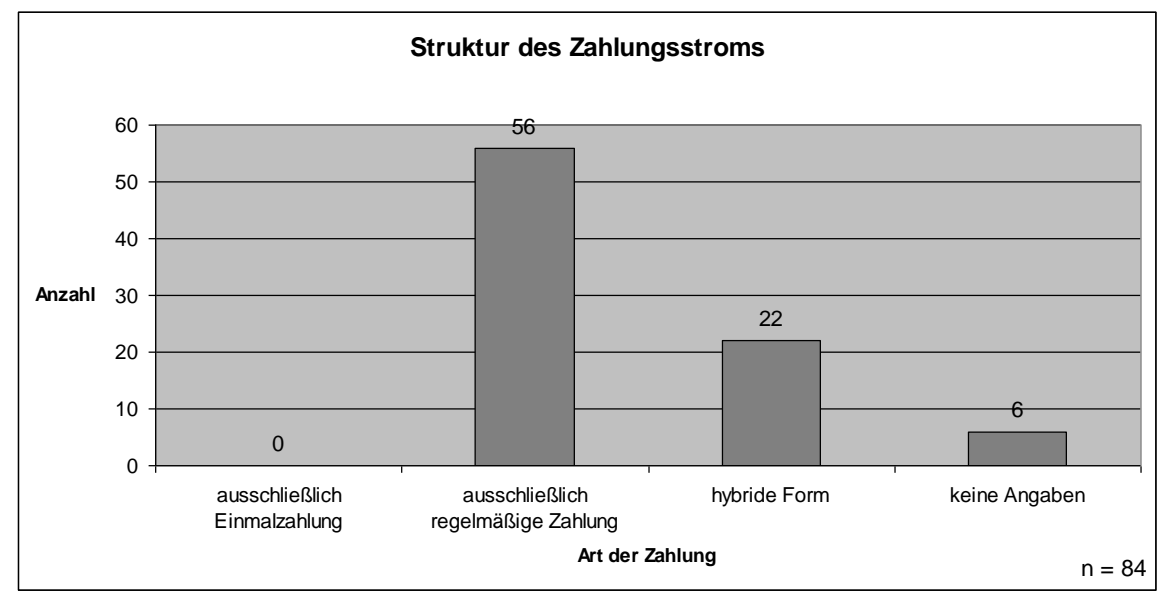

Abbildung 2: Struktur des Zahlungsstroms in Bezug auf Einmal- und/oder regelmäßige Zahlung

\section{Preisdifferenzierung}

Im Folgenden betrachten wir die Preisdifferenzierung zweiten Grades. Sie ist zum einen in der Praxis weit verbreitet, zum anderen ist sie aufgrund der Selbstselektionsmöglichkeit der Kunden ein Teil des an den Kunden zu kommunizierenden Preismodells und daher auch häufig auf den Seiten der SaaS-Anbieter dargestellt.

Sowohl regionen- als auch personenbezogene Preisdifferenzierung wird von Anbietern nicht oder nur in sehr begrenztem Umfang über die Webseite kommuniziert, da hierbei keine Wahlmöglichkeit der Kunden vorliegt und die Kenntnis dieser Form der Preisdifferenzierung bei Kunden möglicherweise eher zu Verärgerungen führen würde.

Die nachfolgende Abbildung 3 zeigt die Ausprägungen der Preisdifferenzierung zweiten Grades in unserer Stichprobe. Durch die Möglichkeit der mehrdimensionalen Preisdifferenzierung (Skiera und Spann 2002, S. 279), d. h. die gleichzeitige Differenzierung nach unterschiedlichen Dimensionen, sind Mehrfachzuordnungen zulässig.

Besonders ausgeprägt ist die mengenbezogene Preisdifferenzierung mit 63 von 75 betrachteten Produkten. So sinkt z. B. der Preis je Nutzer mit steigender Anzahl der Anwender. Ebenfalls verbreitet ist die leistungsbezogene Preisdifferenzierung, bei der Kunden beispielsweise unter Produktvarianten mit unterschiedlichem Funktionsumfang zu unterschiedlichen Preisen wählen können. Vergleichsweise selten konnten wir eine zeitbezogene Preisdifferenzierung feststellen, bei der die Preise z. B. abhängig von der Tages- oder Jahreszeit variieren. 


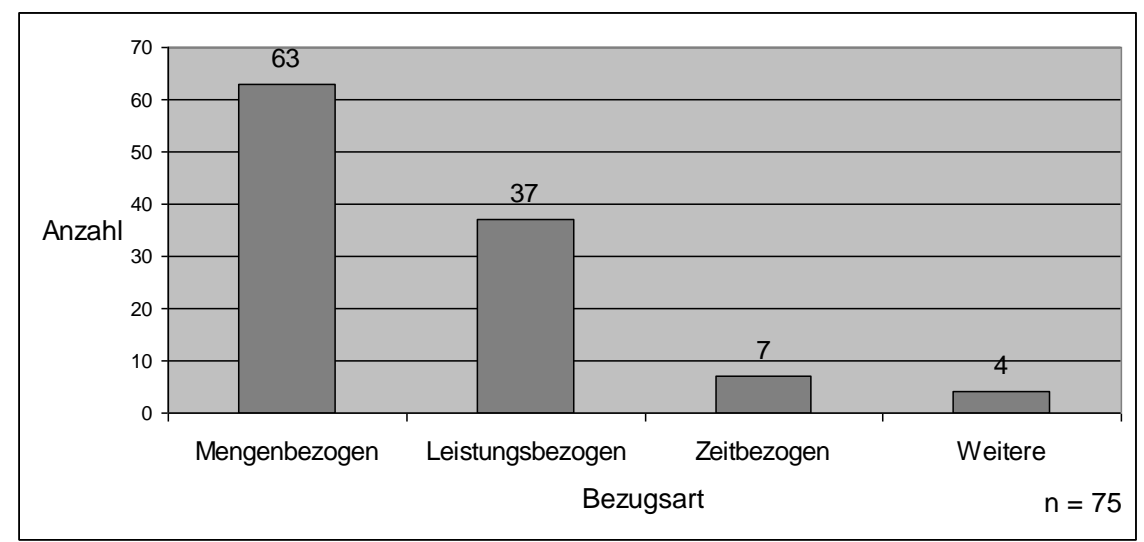

Abbildung 3: Preisdifferenzierung zweiten Grades (Mehrfachzuordnungen sind zulässig)

Im Weiteren betrachten wir den Aspekt der leistungsbezogenen Preisdifferenzierung näher, der auch als Versioning bezeichnet wird (Varian 1997). Laut Informationen der Webseiten der SaaS-Anbieter gibt es bei knapp der Hälfte der betrachteten SaaS-Lösungen mehrere Versionen, unter denen die Kunden wählen können.

Unter den 37 SaaS-Lösungen mit Angaben zur Anzahl der Produktversionen existieren 15 Anwendungen mit genau drei Versionen; dies ist somit - wenn auch nur knapp - die Versionsanzahl mit der häufigsten Nennung. Eine Wahl von drei Versionen wird auch von Varian (1997, S. 200) für Informationsgüter empfohlen. Aufgrund von Extremeness Aversion, der Abneigung gegen Extremes, fällt bei drei Produktvarianten die Entscheidung des Kunden häufig zugunsten der mittleren Preiskategorie. Daher kann es für Anbieter lohnenswert sein, eine Premiumversion zum Produktportfolio hinzuzufügen. Somit wird die Wahl des Kunden auf die mittlere Variante gelenkt, die im Fall von lediglich zwei Versionen die teure der beiden Produktvarianten dargestellt hatte.

\section{Bemessungsgrundlage}

Bei den Bemessungsgrundlagen wird zwischen nutzungsabhängigen und nutzungsunabhängigen Einheiten unterschieden. Nutzungsabhängige Bemessungsgrundlagen orientieren sich an der tatsächlichen Nutzung der Software durch den Kunden (Buxmann et al. 2008a, S. 13). Die Bemessungsgrundlage wird für jede Preiskomponente festgelegt. Daher bestimmt ein Anbieter zunächst die Anzahl der Preiskomponenten. Die Untersuchung der Preiskomponenten ergab, dass 23 Prozent der SaaS-Lösungen lediglich eine Preiskomponente verwenden. Bei 35 Prozent der Produkte enthält das Preismodell zwei Preiskomponenten. Weitere 32 Prozent der Produkte verfügen über drei bis sechs Preiskomponenten. 
Die Analyse aktuell verwendeter Bemessungsgrundlagen zeigt, dass die Mehrheit der Preismodelle auf nutzungsunabhängigen Einheiten basiert (siehe Abbildung 4).

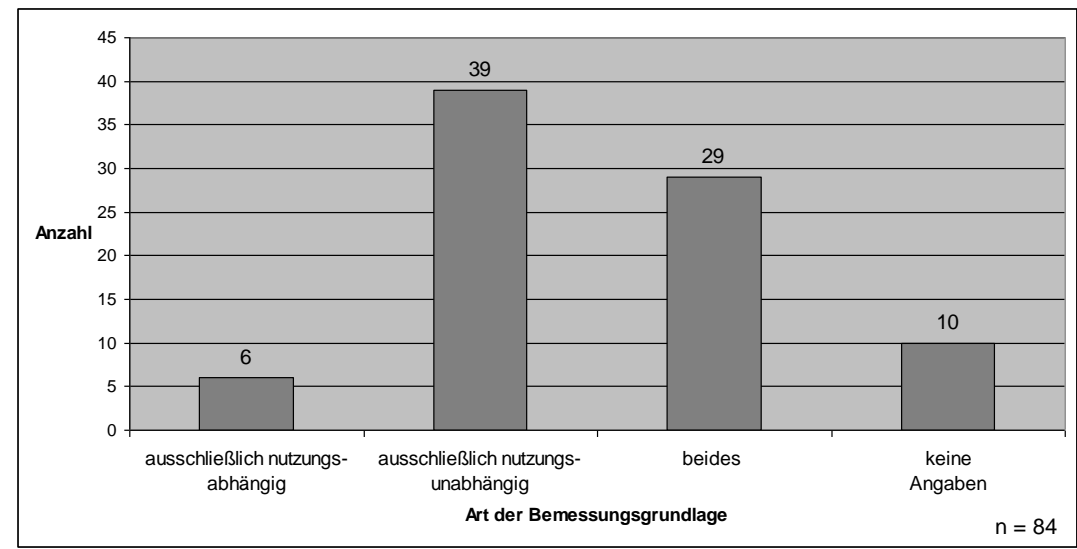

Abbildung 4: Nutzungsabhängige und nutzungsunabhängige Bemessungsgrundlagen

Unter den nutzungsunabhängigen Bemessungsgrundlagen dominieren mit 78 Prozent die User-basierten Preismodelle. Unter ihnen sind 20 Prozent an eine Mindestanzahl an Nutzern gebunden. Neben der klassischen Einheit User konnte in wenigen Fällen eine Bepreisung nach Concurrent User festgestellt werden. 13 Prozent der Produkte mit nutzungsunabhängiger Bemessungsgrundlage basierten auf einer unternehmensweiten Lizenz.

Preismodelle mit ausschließlich nutzungsabhängigen Bemessungsgrundlagen konnten mit 6 von 84 SaaS-Produkten relativ selten beobachtet werden. Häufiger ist hier eine Kombination aus nutzungsabhängigen und nutzungsunabhängigen Bemessungsgrundlagen (29 Produkte). Bei den nutzungsabhängigen Einheiten wird insbesondere auf Transaktionsbasis bepreist. Hierzu zählen u. a. Preise je Buchung, Gehaltsabrechnung, durchgeführte Prüfung, Bestellung oder Zollanmeldung. Ebenso wird auch der Speicherbedarf als nutzungsabhängige Bemessungsgrundlage herangezogen. Dies trat allerdings in den von uns untersuchten Fällen nur als Größe auf, die nach dem Überschreiten einer bestimmten Inklusivmenge berechnet wird, nicht jedoch ab der ersten Einheit.

Weiterhin ist auffällig, dass nutzungsunabhängige Bemessungsgrundlagen fast ausschließlich obligatorisch für den Kunden sind, während nutzungsabhängige Einheiten in vielen Fällen optional vom Kunden gewählt werden können.

Insgesamt zeigt sich, dass der Einsatz von nutzungsabhängigen Bemessungsgrundlagen sich bisher nicht durchgesetzt hat, sondern weiterhin vorwiegend User-basierte Modelle verbreitet sind. In Kapitel 4 gehen wir auf dieses Thema näher ein und untersuchen im Rahmen einer Fallstudie die Zahlungsbereitschaften für nutzungsabhängige und -unabhängige Bemessungsgrundlagen. 

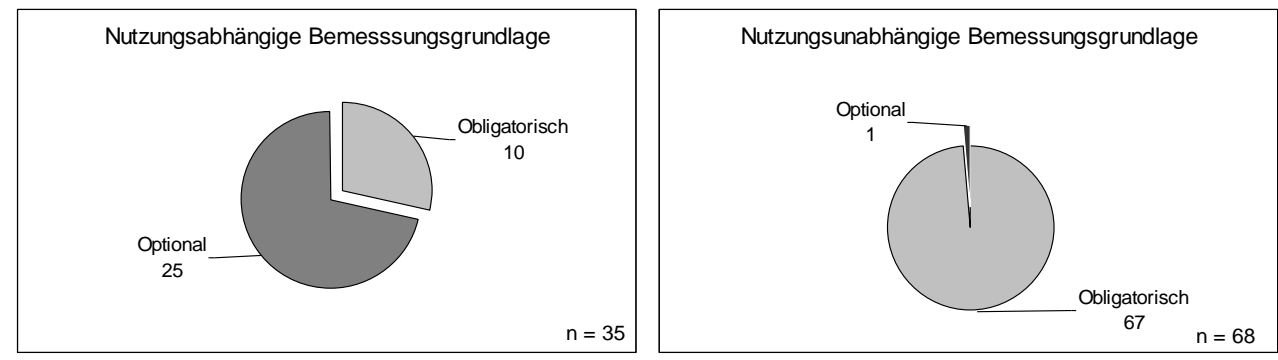

Abbildung 5: Optionale und obligatorische Bemessungsgrundlagen

\section{Fallstudie zum Vergleich nutzungsabhängiger und nutzungsunabhängiger Preismodelle}

Im Folgenden wollen wir die Fragestellung einer nutzungsabhängigen bzw. nutzungsunabhängigen Bepreisung von SaaS-Lösungen anhand eines Beispiels näher erläutern. Insbesondere soll untersucht werden, ob die häufig vertretene These zutrifft, dass das SaaS-Modell besonders für eine nutzungsabhängige Bepreisung geeignet ist (z. B. Kittlaus und Clough 2009, S. 59; Choudhary 2007).

Vor diesem Hintergrund haben wir mit einem Anbieter statistischer Anwendungssoftware für den B2B-Bereich eine Fallstudie durchgeführt. Dieser Softwareanbieter plant eine Applikation, die er zur Zeit als on-Premise-Variante bereitstellt, in Zukunft als SaaS-Lösung anzubieten. In diesem Zusammenhang stellt sich die Frage, welches Preismodell nach der Umstellung gewählt werden soll.

\subsection{Methodik und Datenbasis}

Im Anschluss an die in Kapitel 3 beschriebene Untersuchung wurde im Februar und März 2009 eine telefonische Kundenbefragung durchgeführt. In diesem Zusammenhang wurde das Price Sensitivity Meter (PSM) von van Westendorp (van Westendorp 1976) eingesetzt, um die Zahlungsbereitschaften der Kunden für eine SaaS-Lösung im nutzungsabhängigen sowie nutzungsunabhängigen Fall zu vergleichen. Aufgrund des geringen Umfangs der Stichprobe und der Möglichkeit Gründe für die Entscheidungen zu erfahren, haben wir uns gegen ähnliche Verfahren, wie beispielsweise die Conjoint-Analyse, entschieden.

Bei der van Westendorp-Methode handelt es sich um eine Form der direkten Kundenbefragung, bei der den Befragten vier Fragen zu einem vorab definierten Produkt gestellt werden. Diese Fragen betreffen die Einschätzung des Probanden bezüglich der vier Preispunkte: „günstig“, „teuer“, „zu teuer“ sowie „,zu billig“, wenn er nach einer Preisvorstellung für das Produkt gefragt wird. Die Antworten der einzelnen Teilnehmer werden anschließend aggregiert und als Kurven in einem Diagramm visualisiert. Durch die Schnittpunkte der vier resultierenden Kurven 
wird eine Spanne beschrieben, innerhalb derer der Preis für das angebotene Produkt liegen sollte. ${ }^{2}$ Damit zielt das PSM nicht darauf ab, eine konkrete PreisAbsatz-Funktion zu ermitteln. Vielmehr liegt die Zielsetzung in der Ermittlung einer „akzeptablen Preisspanne“ (Lock 1998; S. 507) für ein innovatives Produkt, für das noch keine Preisvorstellung existiert.

Die Stichprobe bestand aus 28 Kunden dieses Softwareanbieters, die das vorgestellte SaaS-Produkt bereits als on-Premise-Variante nutzen. Daher kennen die Kunden sowohl die Funktionalität der Anwendung als auch die im aktuellen Lizenzierungsmodell anfallenden Kosten.

Hinsichtlich der Unternehmensgröße besteht die Stichprobe zu rund 38 Prozent aus kleinen und mittleren Unternehmen mit einem Jahresumsatz von weniger als 500 Mio. $€$ und zu 62 Prozent aus großen Unternehmen mit mehr als 500 Mio. $€$ Jahresumsatz. Die Unternehmen entstammen vorwiegend der Automobil- und Zulieferindustrie im deutschsprachigen Raum. Im Folgenden gehen wir auf die Ergebnisse der durchgeführten Befragung ein.

\subsection{Ergebnisse}

Die van Westendorp-Methode wurde im Rahmen der Befragung zweimal eingesetzt. Im ersten Fall wurde für das Preismodell der beschriebenen SaaSAnwendung die nutzungsunabhängige Bemessungsgrundlage des Concurrent User gewählt. Mit zwölf von 28 Befragten machten rund 43 Prozent der Probanden Angaben zu allen vier abgefragten Preispunkten. Die Ergebnisse dieser Untersuchung werden in Abbildung 6 illustriert. Das PSM liefert - wie oben beschrieben eine Preisspanne, innerhalb derer der Preis für die Anwendung festgelegt werden sollte. In diesem Fall liegt die Empfehlung zwischen dem „Point of Marginal Cheapness“ (PMC) und dem „Point of Marginal Expensiveness“ (PME); also zwischen 5,46 € und 24,10 € pro Monat und Concurrent User.

Die parallel durchgeführte Erhebung der Zahlungsbereitschaften im nutzungsabhängigen Fall lieferte eine deutlich geringere Antwortquote. Da die Software u. a. die Anfertigung von statistischen Berichten ermöglicht, wurde als nutzungsabhängige Bemessungsgrundlage die Transaktion ,angefertigter Bericht“ gewählt. Lediglich 14 Prozent (vier der 28 Teilnehmer) nannten konkrete Werte für alle vier abgefragten Preispunkte.

Dennoch lassen sich aus den Ergebnissen erste Erkenntnisse hinsichtlich der geringen Bedeutung nutzungsabhängiger Preismodelle ableiten. Ergänzend zu den konkreten Preispunkten konnten durch die telefonische Befragung Hintergründe für die fehlende Einschätzungsfähigkeit der Teilnehmer in die Auswertung einbezogen werden: Der Mehrheit der Befragten ist die tatsächliche Nutzungsintensität ihrer eingesetzten Software nicht bekannt.

${ }^{2}$ Für die Bedeutung der einzelnen Schnittpunkte siehe u. a. Simon 2008, S. 175. 


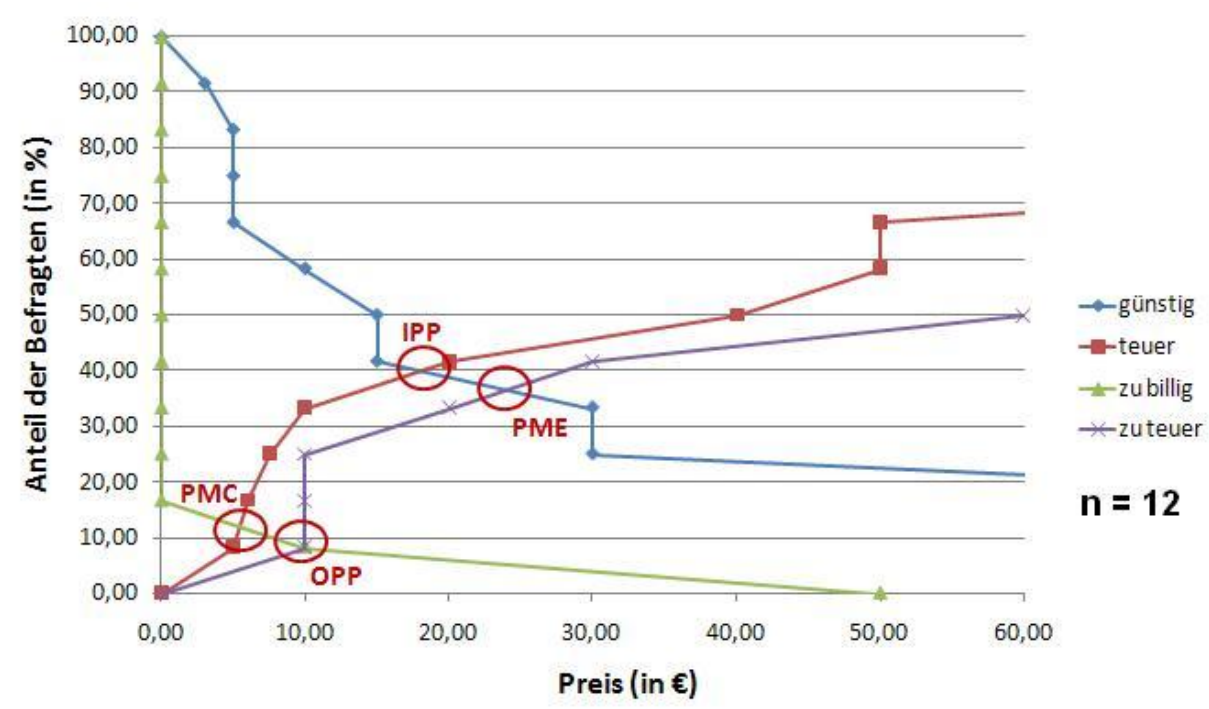

\section{Abbildung 6: Ergebnisse des PSM im nutzungsunabhängigen Fall}

Da die Intensität jedoch im Falle einer nutzungsabhängigen Bemessungsgrundlage die Kosten bestimmt, ist es diesen Kunden nicht möglich, eine Einschätzung der Zahlungsbereitschaft anzugeben.

Als weiteres Problem stellte sich die Auswahl der konkreten Bemessungsgrundlage - wie hier in Form des Berichts - heraus. Aufgrund der Vielfältigkeit der Software und dem daraus resultierenden heterogenen Nutzungsverhalten der Anwender, bestanden unterschiedliche Auffassungen über eine geeignete nutzungsabhängige Bemessungsgrundlage.

Auch unter den Antwortenden wurden unerwartete Ergebnisse erzielt. So bestand zwischen den Einschätzungen zweier Befragter zum „günstig“-Preispunkt ein Unterschied von einem Faktor größer 1000, was auf eine sehr heterogene Zahlungsbereitschaft der Kunden hinweist.

\section{$5 \quad$ Fazit und Ausblick}

Um der Frage nachzugehen, ob SaaS-Lösungen insbesondere durch ihre Preisgestaltung für Kunden und Anbieter interessant sind, haben wir 80 SaaS-Anbieter im B2B-Umfeld anhand ihrer Webseiten analysiert.

Derzeitige Diskussionen über die nutzungsabhängige Preisgestaltung von SaaSLösungen konnten durch unsere Ergebnisse nicht bestätigt werden. Bei den Bemessungsgrundlagen kommen überwiegend nutzungsunabhängige Einheiten zum Einsatz. Nutzungsabhängige Bemessungsgrundlagen wie Preise je durchgeführte Transaktion hingegen sind für Kunden meist optional. 
Die Analyse der Zahlungsbereitschaften für nutzungsabhängige Bemessungsgrundlagen mit Hilfe der van Westendorp-Methode im Rahmen einer Fallstudie hat ergeben, dass die Mehrheit der Kunden ihre Nutzungsintensität nicht kennt und daher keine Aussage treffen kann. Unter den Kunden, die eine konkrete Zahlungsbereitschaft nannten, variierte die Zahlungsbereitschaft um den Faktor 1000. Aus Anbieterperspektive ist diese ausgeprägte Heterogenität der Zahlungsbereitschaften besonders ungünstig. Um einerseits viele Kunden zu erreichen und andererseits deren Zahlungsbereitschaft bestmöglich abzuschöpfen, ist eine homogene Struktur der Zahlungsbereitschaften mit einer geringen Varianz erforderlich.

Generell bietet sich die Preisdifferenzierung zum Abschöpfen unterschiedlicher Zahlungsbereitschaften an. Dies dürfte jedoch in diesen Dimensionen schwierig umzusetzen sein. Weiterhin kommt hinzu, dass die Preisgestaltung für SaaSProdukte vermutlich aufgrund des Vertriebs über Internet tendenziell transparenter sein wird als beispielsweise bei on-Premise-Software. Dies erschwert insbesondere Preisdifferenzierung dritten Grades.

Die Ergebnisse der Untersuchung zeigen, dass eine nutzungsabhängige Bemessungsgrundlage - auch wenn sie für SaaS als prädestiniert angesehen wird - ungeeignet sein kann, wenn die ungeschickte Wahl der Bemessungsgrundlage zu einer stark heterogenen Struktur der Zahlungsbereitschaften führt.

Allerdings ist zu beachten, dass die Erhebung der Zahlungsbereitschaften unter potenziellen SaaS-Kunden eines spezifischen Softwareprodukts durchgeführt wurde. Von Interesse sind vergleichbare Erhebungen unter SaaS-Produkten aus anderen Bereichen, wie beispielsweise einer ERP- oder CRM-Software sowie die allgemeine Analyse des Zusammenhangs zwischen der Verteilung von Zahlungsbereitschaften und der Ausgestaltung des Preismodells.

$\mathrm{Da}$ in der vorliegenden Studie nur drei der im zweiten Kapitel beschriebenen sechs Parameter der Software-Preisgestaltung analysiert werden konnten, besteht insbesondere für die verbleibenden Parameter Preisbildung, Preisbündelung und dynamische Preisstrategien Forschungsbedarf. Außerdem bestehen noch offene Fragen hinsichtlich der erzielbaren Profitabilität der SaaS-Anbieter durch Anwendung dieser Preismodelle.

\section{Literatur}

Benlian A, Hess T, Buxmann P (2009) Treiber der Adoption SaaS-basierter Anwendungen - Eine empirische Untersuchung auf Basis verschiedener Applikationstypen. WIRTSCHAFTSINFORMATIK 51(5):414-428.

Bontis N, Chung H (2000) The evolution of software pricing: From box licenses to application service provider models. Electronic Networking Applications and Policy 10(3):246-255. 
Buxmann P, Diefenbach H, Hess T (2008a) Die Softwareindustrie: Ökonomische Prinzipien, Strategien, Perspektiven. Springer, Berlin.

Buxmann P, Hess T, Lehmann S (2008b) Software as a Service. WIRTSCHAFTSINFORMATIK 50(6):500-503.

Choudhary V (2007) Software as a Service: implications for investment in software development. Proceedings of the 40th Hawaii International Conference on System Sciences (HICSS 2007), Hawaii, 2007.

Cusumano MA (2007) The changing labyrinth of software pricing. Communications of the ACM 50(7):19-22.

Kittlaus HB, Clough PN (2009) Software product management and pricing. Key success factors for software organizations. Springer, Berlin.

Lehmann S, Buxmann P (2009) Preisstrategien von Softwareanbietern. WIRTSCHAFTSINFORMATIK 51(6), im Druck..

Lock D (1998) The Gower handbook of management. 4. Auflage. Gower Publishing, Hampshire.

Simon H, Fassnacht M (2008) Preismanagement. 3. Auflage. Gabler, Wiesbaden.

Skiera B, Spann M (2002) Preisdifferenzierung im Internet. In: Schögel M, Tomczak T, Belz C (Hrsg.) Roadm@p to E-Business. Thexis, St. Gallen, 270284.

van Westendorp PH (1976) NSS-Price Sensitivity Meter: a new approach to study consumer perception of prices. Venice ESOMAR Congress, Amsterdam, 1976, 139-167.

Varian HR (1997) Versioning information goods. In: Kahin B, Varian HR (Hrsg.) Internet publishing and beyond: the economics of digital information and intellectual property. MIT, Cambridge, 190-202. 